\title{
Urological outcomes in nonagenarians with prostate cancer
}

Josh White; Jesse Ory; Heather Morris; Ricardo A. Rendon; Ross Mason; Ashley Cox Department of Urology, Dalhousie University, Halifax, NA, Canada

Cite as: White J, Ory J, Morris H, et al. Urological outcomes in nonagenarians with prostate cancer. Can Urol Assoc J 2021 January 4; Epub ahead of print. http://dx.doi.org/10.5489/cuaj.6805

Published online January 4, 2021

$* * *$

\section{Abstract}

Introduction: Nonagenarians represent a growing patient population. Herein, we report on the largest cohort of Canadian nonagenarian patients, to our knowledge, with prostate cancer.

Methods: A retrospective chart of 44 nonagenarian men diagnosed with localized or metastatic prostate cancer between 2006 and 2019 was performed. Diagnoses were based on pathological specimens or the presence of a high prostate-specific antigen (PSA $>20$ ) or abnormal digital rectal exam (DRE) in the setting of metastatic disease on imaging. Patient demographics, presenting complaints, and treatments required were included in the analysis. A descriptive statistical analysis was performed.

Results: The median patient age at time of referral was 91.1 years (interquartile range [IQR] 90.2-92.9). The median PSA at time of referral was 54.0 (IQR 18.2-142.6). Metastatic disease was present in $55 \%$ of patients at time of diagnosis $(n=24)$. Most patients required at least one urological intervention $(\mathrm{n}=35)$. There were $56.8 \%$ of patients who received androgen deprivation therapy (ADT) as part of their treatment regime $(n=25)$. Half $(50 \%)$ of patients were managed with androgen receptor axis-targeted agents (ARAT), as well as ADT $(n=22)$. Five patients (11.4\%) underwent surgical castration. Death due to any cause was noted in $52.3 \%$ of patients $(\mathrm{n}=23)$ throughout the study period, with the median age at death being 94.4 years (IQR 92.397.0). Death due to prostate cancer was noted in $18.2 \%$ of patients $(n=8)$.

Conclusions: This study highlights common presenting complaints for nonagenarian patients with prostate cancer and that many require urological intervention despite advanced age. Future studies should address patient-reported quality of life outcomes in the nonagenarian population with prostate cancer. 


\section{Introduction}

With modern advances in medicine, physicians and healthcare professionals face a unique patient population as life expectancy increases. The life expectancy of Canadians in 2016 was 82.3 years, representing a $15.4 \%$ increase since $1960 .{ }^{1}$ The nonagenarian and centenarian population are among the fastest growing age groups in Canada and the nonagenarian population is growing steadily worldwide; in 2050, world population projections estimate 76.71 million people aged 90 years or older. ${ }^{2,3}$ Prostate cancer is the most common non-cutaneous malignancy in men and its prevalence increases with age. ${ }^{4}$ Nonagenarians are largely underrepresented within clinical trials and there is a paucity of literature to assist physicians in making clinical decisions regarding their care. In order to provide effective treatment for this emergent patient group it is important to obtain data about nonagenarians with prostate cancer.

Prostate cancer is generally diagnosed in younger men based on routine prostate specific antigen (PSA) screening or an abnormal DRE leading to biopsies. Canadian Urological Association (CUA) guidelines suggest limiting prostate cancer screening with PSA to men with a life expectancy of greater than 10 years. ${ }^{5}$ The National Comprehensive Cancer Network (NCCN) guidelines endorse observing localized disease in patients with a life expectancy of less than 5-10 years, depending on risk group, and considering palliative ADT with or without radiation for metastatic disease. ${ }^{6}$ Clinicians would expect for nonagenarians to be diagnosed more commonly with metastatic disease. Both conservative and palliative treatment options may have significant side effects or result in urological issues that impact quality of life in the nonagenarian population.

This descriptive study aims to assess the interventions and treatment modalities used by urologists in a tertiary care centre to manage nonagenarian patients with prostate cancer. We hypothesized that the majority of nonagenarian patients would be managed with observation or palliative androgen deprivation therapy (ADT). We also hypothesized that there would be a minority of nonagenarians who died of prostate cancer. To the best of our knowledge, this study represents the first reported dataset of Canadian nonagenarian patients with prostate cancer.

\section{Methods}

This project was approved by our local ethics board. The initial nonagenarian database was created by evaluating both inpatient and outpatient referrals made to a urologist at our tertiary care centre between 2006 and 2019. Referrals were identified through billing codes in the electronic medical record (EMR). Patients were included if there was a new diagnosis of prostate cancer as a nonagenarian or if the patient was referred with existing prostate cancer with new or progressive symptoms for management by urology.

Diagnoses were based on pathological specimens or the presence of a high PSA and/or an abnormal DRE in the setting of metastatic disease on imaging. For men diagnosed exclusively on PSA, the inclusion cutoff was $50 \mathrm{ng} / \mathrm{ml}$. If an elevated PSA was in conjunction with physical 
exam or diagnostic imaging findings consistent with prostate cancer, the PSA cutoff was 20 $\mathrm{ng} / \mathrm{ml}$. Patient demographics and comorbidities were included in the analysis, as well as presenting complaints and treatments administered. Comorbidities evaluated included Class I comorbidities as per the Charlson Comorbidity Index. ${ }^{7}$ Androgen deprivation therapy (ADT) included luteinizing hormone-releasing (LHRH) agonists and antagonists. Surgical castration was recorded separately. Androgen receptor axis-targeted (ARAT) agents were evaluated separately. Skeletal related events included: pathological fractures, spinal cord compression or severe bony pain requiring radiation or surgery to the bone. ${ }^{8}$

Cause of death was determined by evaluating death certificates. Patients with a questionable diagnosis of prostate cancer were excluded from the analytical sample. Medians and interquartile range (IQR) were reported for continuous variables. Frequencies and proportions were reported for categorical variables.

\section{Results}

A retrospective chart review of 44 nonagenarian men diagnosed with localized or metastatic prostate cancer between 2006 and 2019 was performed. 36 of these were outpatient referrals with 8 being inpatient referrals. The median patient age at time of referral was 91.1 years (IQR 90.292.9). $54.5 \%$ of patients presented with metastatic disease $(n=24)$. The most common method of diagnosis was by elevated PSA $(n=21)$. The median PSA at time of referral was 54.0 (IQR 18.2 142.6). The median PSA for patients presenting with metastatic disease was 130.0 (IQR 57.6 322.6). 18.2\% of patients were diagnosed by a prostate biopsy $(n=8) .20 .5 \%$ of patients were diagnosed based off a TURP specimen $(n=9)$. The median PSA for patients diagnosed by prostate biopsy was 35.6 (IQR $16.8-77.3$ ). $75 \%$ of the patients who had a prostate biopsy diagnosis had metastatic disease at the time of presentation $(n=6)$. The median PSA for patients diagnosed by TURP specimen was 52.5 (IQR 12.2 - 73). 44.4\% of patients diagnosed by TURP specimen had metastatic disease at the time of presentation $(n=4) .2$ patients were diagnosed on the basis of an abnormal DRE. $72.7 \%$ of patients had one or more Class I comorbidities as per the Charlson comorbidity index $(n=32)$. Coronary artery disease (CAD) was the most common comorbidity identified, found in $56.8 \%$ of patients $(n=25) .29 .5 \%$ of patients had concurrent malignancy $(n=13) .23 .1 \%$ of patients with concurrent malignancy had a known history of urothelial carcinoma of the bladder $(n=3) .59 .1 \%$ of patients were living independently at the time of referral $(n=26)$. A summary of demographic data for the entire cohort can be found in Table 1.

The most common presenting symptom patients experienced was urinary retention, which was defined as urinary retention requiring catheterization, with $63.6 \%$ of patients presenting with urinary retention at the time of referral $(n=28)$. Skeletal related events occurred in $29.5 \%$ of patients $(n=13)$ with spinal cord compression occurring in $4.5 \%(n=2)$. Both of the patients presenting with spinal cord compression received urgent radiation. $43.2 \%$ of patients 
presented with gross hematuria $(n=19)$ though only $13.6 \%$ of patients received an evacuation of clots $(\mathrm{EoC}, \mathrm{n}=6)$. Only two of the patients who received an EoC required a blood transfusion. $13.6 \%$ of the entire cohort required at least one blood transfusion during their treatment course $(n=6)$. The overwhelming majority of patients required at least one urological intervention $(\mathrm{n}=35)$. Cystoscopy and catheter insertion were the most common interventions required at $72.7 \%(n=32)$ and $75.0 \%$ respectively $(n=33)$. Table 2 shows presenting symptoms and urological procedures among the entire cohort.

There were $56.8 \%$ of patients who received ADT as part of their treatment regime $(n=25) .50 \%$ of patients were managed with ARAT as well as ADT $(n=22) .11 .4 \%$ of patients underwent surgical castration $(n=5)$. One patient received docetaxal. $25.0 \%$ of patients obtained a referral to radiation oncology $(n=11) .15 .9 \%$ patients received palliative radiation as a part of their treatment course $(n=7)$. Patients treated with palliative radiation all presented with skeletal related events. $18.2 \%$ of patients received a referral to medical oncology. $20.5 \%$ of patients received a referral to palliative care $(n=9)$. Table 3 shows the treatment modality the patient cohort received for management of prostate cancer.

Twenty-three men (52.5\%) died during the study period at a median age of 94.4 years (IQR 92.3 - 97.0). The median time to death from time of referral was 22.7 months (IQR $10.4-$ $39.1)$. Eight men $(18.2 \%)$ died of prostate cancer at a median time to death from referral of 14.1 months (IQR 6.6-21.1). 4 of the 8 patients who died of prostate cancer received one or both of ADT and ARAT. 6 of the 8 patients who died of prostate cancer presented with metastatic disease. Of the 24 patients that presented with metastatic disease, 22 received ADT. $62.5 \%$ of patients with metastatic disease died $(n=15)$. Table 4 shows mortality data on our cohort of nonagenarian patients.

\section{Discussion}

Herein, we describe a cohort of nonagenarian patients with prostate cancer managed at our institution. We found that the majority of nonagenarian patients were diagnosed with prostate cancer due to symptomatic disease, which prompted their referral. The management of nonagenarians without metastatic disease is not controversial, though the management of metastatic disease represents a more challenging setting wherein clinicians must weigh the risks and benefits for treatment. Many of our patients presented with metastatic disease, which helps to explain the large proportion of patients who received ADT. We expect that the number of patients with metastatic disease was likely higher, though was not confirmed with imaging as clinicians may also forgo staging investigations and presume metastatic disease based on PSA alone. Death due to prostate cancer based on cause of death from the death certificates was low in our cohort, though follow-up was limited in many of these patients.

Patients presenting with an elevated PSA represented a surprisingly large percentage of our cohort. It is important that none of the PSA referrals were screening PSA tests, and rather 
patients were experiencing symptoms and a PSA was ordered as part of the work-up. As such, it is difficult to determine which proportion of PSA tests would be considered inappropriate. In our centre, many patients with metastatic disease are managed through the Advanced Prostate Cancer clinic, which is led by urology. This may help to explain the low number of referrals to medical oncology with the relatively large number of patients being managed with ADT and ARAT.

There were a surprisingly high number of patients who underwent prostate biopsies in our cohort. Some of these patients underwent biopsies as part of qualification for clinical trials. One patient underwent a prostate biopsy, which was ordered by the family physician through interventional radiology with subsequent referral to urology. The rationale for the remaining biopsies was unclear.

The management of organ confined prostate cancer in nonagenarians is not controversial. The NCCN advocates against the use of ADT in patients with a life expectancy of 5-10 years. In the largest series on octogenarian and nonagenarian patients with organ confined prostate cancer, Dell'Oglio et al. found that the cancer specific mortality was only $19.7 \% .{ }^{10}$ They advocate against the use of ADT in those with organ-confined disease, as only 3.1\% of men aged 90 and above will live for 10 years. ${ }^{10}$ This study, however, did not include patients with metastatic disease, where ADT is a mainstay of treatment. ${ }^{12}$

Metastatic prostate cancer presents a unique and challenging situation for urologists when making decisions for the appropriate care of patients with advanced age, as ADT is not a benign form of treatment. Patient symptoms, baseline function and life expectancy must be considered. Urologists, however, have been shown to be poor estimators of life expectancy in relation to prostate cancer management. ${ }^{9}$ This further adds to the complexity of weighing the risks and benefits of treatment of systemic therapy in the nonagenarian population. None of our patients lived longer than 10 years from the time of referral. Nearly $60 \%$ of patients lived independently at the time of referral, indicative of a relatively high level of function. Unfortunately, collective prospective data to evaluate quality of life outcomes is unlikely to occur in this patient population. It is important to note that the issue is not that urologists expect prostate cancer to take a distinctive form in the nonagenarian population.

This is a retrospective analysis and therefore limited by an inherent selection bias. All of the patients in our cohort were referred to our tertiary care centre, thereby excluding patients managed in the community. Moreover, nearly half of the patients were referred to our Advanced Prostate Cancer clinic, resulting in a referral bias. As such, our patient cohort may have more advanced disease than many nonagenarians with prostate cancer. The natural progression of prostate cancer may in part account for the large proportion of patients presenting with metastatic disease. Recommendations for treatment should not be drawn from our study, as the results are purely descriptive. Another limitation to our study is the size of our cohort. While there were 
only 44 patients, this represents the largest cohort of nonagenarian patients with prostate cancer in Canada.

\section{Conclusions}

As life expectancy increases, urologists can expect for the number of referrals on patients with advanced age and prostate cancer to increase. Nonagenarian patients with prostate cancer present with multiple urological complaints, many of which require intervention. Our patients were largely managed with ADT +/- ARAT. Future studies assessing patient-reported quality of life outcomes in the nonagenarian population with prostate cancer would aid in the determination of optimal management strategies for this unique patient population. 


\section{References}

1. World Bank: World development indicators: Canadian life expectancy. 2018. Available at: https://data.worldbank.org/indicator/SP.DYN.LE00.MA.IN?locations=CA.

2. Decady Y and Greenberg L: Ninety years of change in life expectancy. Stat. Can.: 10.

3. United Nations, Department of Economic and Social Affairs and Population Division: World population prospects Highlights, 2019 revision Highlights, 2019 revision.; 2019.

4. Canadian Cancer Statistics Advisory Committee: Canadian cancer statistics 2019. Can. Cancer Soc. 2019.

5. Rendon RA, Mason RJ, Marzouk K, et al: Canadian Urological Association recommendations on prostate cancer screening and early diagnosis. Can. Urol. Assoc. J. 2017; 11: 298-309.

6. Mohler JL, Armstrong AJ, Bahnson RR, et al: Prostate Cancer, Version 1.2016. J. Natl. Compr. Cancer Netw. JNCCN 2016; 14: 19-30.

7. Brusselaers $\mathrm{N}$ and Lagergren J: The Charlson Comorbidity Index in Registry-based Research. Methods Inf. Med. 2017; 56: 401-406.

8. So A, Chin J, Fleshner N, et al: Management of skeletal-related events in patients with advanced prostate cancer and bone metastases: Incorporating new agents into clinical practice. Can. Urol. Assoc. J. J. Assoc. Urol. Can. 2012; 6: 465-470.

9. Leung KMYB, Hopman WM and Kawakami J: Challenging the 10-year rule: The accuracy of patient life expectancy predictions by physicians in relation to prostate cancer management. Can. Urol. Assoc. J. J. Assoc. Urol. Can. 2012; 6: 367-373.

10. Dell'Oglio P, Bishr M, Boehm K, et al: Survival Outcomes in Octogenarian and Nonagenarian Patients Treated with First-line Androgen Deprivation Therapy for Organconfined Prostate Cancer. Eur. Urol. Focus 2018; 4: 834-841.

11. Valdivieso R, Boehm K, Meskawi M, et al: Patterns of use and patient characteristics: brachytherapy for localized prostate cancer in octo- and nonagenarians. World J. Urol. 2015; 33: 1985-1991.

12. Cannata H Dara, Kirschenbaum C Alexander and Levine C Alice: Androgen Deprivation Therapy as Primary Treatment for Prostate Cancer. J. Clin. Endocrinol. Metab. 2012; 97 : 360-365. 
Figures and Tables

\begin{tabular}{|c|c|}
\hline Variables & Overall \\
\hline \multicolumn{2}{|l|}{ Age of referral (yrs) } \\
\hline Median (IQR) & $91.1(90.2-92.9)$ \\
\hline \multicolumn{2}{|l|}{ PSA at time of referral } \\
\hline Median (IQR) & $54.0(18.2-142.6)$ \\
\hline \multicolumn{2}{|c|}{ Presentation with metastatic disease, n (\%) } \\
\hline Metastatic & $24(54.5)$ \\
\hline \multicolumn{2}{|l|}{ Method of diagnosis, $n(\%)$} \\
\hline PSA & $21(47.7)$ \\
\hline TURP & $9(20.5)$ \\
\hline Biopsy & $8(18.2)$ \\
\hline Imaging & $2(4.5)$ \\
\hline DRE & $2(4.5)$ \\
\hline Unknown & $2(4.5)$ \\
\hline \multicolumn{2}{|l|}{ Home environment, n (\%) } \\
\hline Independent living & $26(59.1 \%)$ \\
\hline Non-independent living & $18(40.9 \%)$ \\
\hline \multicolumn{2}{|l|}{ Comorbidities, n (\%) } \\
\hline Coronary artery disease & $25(56.8)$ \\
\hline Other cancer & $13(29.5)$ \\
\hline Dementia & $11(25.0)$ \\
\hline Chronic kidney disease & $8(18.2)$ \\
\hline Diabetes & $8(18.2)$ \\
\hline
\end{tabular}

DRE: digital rectal exam; IQR: interquartile range; PSA: prostate-specific antigen; TURP: transurethral resection of prostate. 


\begin{tabular}{|l|l|}
\begin{tabular}{|l|l|} 
Table 2. Symptoms on presentation to urology and urological \\
procedures performed
\end{tabular} \\
\hline Variables & Overall \\
\hline Urinary symptoms, $n(\%)$ & $28(63.6)$ \\
\hline Urinary retention & $24(54.5)$ \\
\hline Pain & $19(43.2)$ \\
\hline Hematuria & $13(29.5)$ \\
\hline Skeletal related events & $2(4.5)$ \\
\hline Spinal cord compression & \\
\hline Urological procedures required, n (\%) & $33(75.0)$ \\
\hline Catheterization & $32(72.7)$ \\
\hline Cystoscopy & $6(13.6)$ \\
\hline Evacuation of clots & $6(13.6)$ \\
\hline TURP & $1(2.3)$ \\
\hline Nephrostomy tube & $1(2.3)$ \\
\hline Ureteric stent &
\end{tabular}

TURP: transurethral resection of prostate.

\begin{tabular}{|l|c|}
\hline \multicolumn{2}{|l|}{ Table 3. Treatment modality } \\
\hline Treatment modality used, $\mathbf{n}(\%)$ & Overall \\
\hline ADT & $25(56.8)$ \\
\hline ARAT & $22(50.0)$ \\
\hline Watchful waiting & $10(22.7)$ \\
\hline Palliative radiation & $7(15.9)$ \\
\hline Bilateral orchiectomy & $5(11.4)$ \\
\hline Chemotherapy & $1(2.3)$ \\
\hline
\end{tabular}

ADT: androgen deprivation therapy; ARAT: androgen receptor axis-targeted agents; EBRT: external beam radiation therapy; TURP: transurethral resection of prostate. 


\begin{tabular}{|l|c|}
\hline Table 4. Mortality data & Overall \\
\hline Variables & $23(52.3)$ \\
\hline Death due to any cause, $\mathrm{n}(\%)$ & $8(18.2)$ \\
\hline Death due to prostate cancer, $\mathrm{n}(\%)$ & \\
\hline Age of death (years) & $94.4(92.3-97.0)$ \\
\hline Median (IQR) & \\
\hline Time from referral to death - all (months) & $22.7(10.4-39.1)$ \\
\hline Median (IQR) & \\
\hline Time from referral to death - prostate (months) & $14.1(6.6-21.1)$ \\
\hline Median (IQR) &
\end{tabular}

IQR: interquartile range. 